\title{
The Brunn-Minkowski Inequalities for Centroid Body
}

\author{
Jun Yuan, Lingzhi Zhao \\ School of Mathematics and Computer Science, Nanjing Xiaozhuang University, Nanjing, China \\ Email: yuanjun_math@126.com
}

Received August 9, 2012; revised September 22, 2012; accepted October 6, 2012

\begin{abstract}
In [1], the authors established the Brunn-Minkowski inequality for centroid body. In this paper, we give an isolate form and volume difference of it, respectively. Both of these results are strength versions of the original.
\end{abstract}

Keywords: Centroid Body; The Brunn-Minkowski Inequality

\section{Introduction}

The setting for this paper is $n$-dimensional Euclidean space $\mathbb{R}^{n}$. Let $\mathcal{K}^{n}$ denote the set of convex bodies (compact, convex subsets with non-empty interiors). Let $B_{n}$ and $S^{n-1}$ denote the unit ball and unit sphere in $\mathbb{R}^{n}$, respectively. If $K \in K^{n}$, then the support function of $K, h_{K}=h(K, \cdot): S^{n-1} \rightarrow \mathbb{R}$, is defined by

$$
h(K, u)=\max \{u \cdot x: x \in K\}, u \in S^{n-1}
$$

where $u \cdot x$ denotes the standard inner product of $u$ and $x$.

For each compact star-shaped about the origin $K \subset \mathbb{R}^{n}$, denoted by $V(K)$ its $n$-dimensional volume. The centroid body $\Gamma K$ of $K$ is the origin-symmetric convex body whose support function is given by (see [2])

$$
h(\Gamma K, u)=\frac{1}{V(K)} \int_{K}|u \cdot x| \mathrm{d} x,
$$

where the integration is with respect to Lebesgue measure on $\mathbb{R}^{n}$.

Centroid body was attributed by Blaschke and Dupin (see [3,4]), it was defined and investigated by Petty [2]. More results regarding centroid body see [2-7].

For star body $K$ and $L$, let $K \hat{+} L$ denote the harmonic Blaschke addition of $K$ and $L$. In [1], the authors established the following Brunn-Minkowski inequality for centroid body.

Theorem A. Let $K, L$ be star bodies in $\mathbb{R}^{n}$. Then

$$
V(\Gamma(K \hat{+} L))^{\frac{1}{n}} \geq V(\Gamma K)^{\frac{1}{n}}+V(\Gamma L)^{\frac{1}{n}},
$$

the equality holds if and only if $\Gamma K$ and $\Gamma L$ are homothetic.
In this paper, we give two strength versions of (1.3). Our main results are the following two theorems.

Theorem 1.1. Let $K, L$ be star bodies in $\mathbb{R}^{n}$ and $0 \leq \alpha \leq 1$.

$$
\begin{aligned}
& V(\Gamma(K \hat{+} L))^{\frac{1}{n}} \\
& \geq V(\Gamma(\alpha K \hat{+}(1-\alpha))) L^{\frac{1}{n}}+V(\Gamma((1-\alpha) K \hat{+} \alpha L))^{\frac{1}{n}} \\
& \geq V(\Gamma K)^{\frac{1}{n}}+V(\Gamma L)^{\frac{1}{n}},
\end{aligned}
$$

the equality holds if and only if $\Gamma K$ and $\Gamma L$ are homothetic.

Theorem 1.2. Let $K$ and $L$ be star bodies in $\mathbb{R}^{n}$. Ellipsoid $E_{1} \subset K$, and $E_{2} \subset L$ is a homothetic copy of $E_{1}$. Then

$$
\begin{aligned}
& {\left[V(\Gamma(K \hat{+} L))-V\left(\Gamma\left(E_{1} \hat{+} E_{2}\right)\right)\right]^{\frac{1}{n}}} \\
& \geq\left[V(\Gamma K)-V\left(\Gamma E_{1}\right)\right]^{\frac{1}{n}}+\left[V(\Gamma L)-V\left(\Gamma E_{2}\right)\right]^{\frac{1}{n}},
\end{aligned}
$$

the equality holds if and only if $\Gamma K$ and $\Gamma L$ are homothetic and

$$
\left(V(\Gamma K), V\left(\Gamma E_{1}\right)\right)=\mu\left(V(\Gamma L), V\left(\Gamma E_{2}\right)\right),
$$

where $\mu$ is a constant.

Remark. Let $\alpha=1$ or $\alpha=0$ in Theorem 1.1, or let $E_{1}=E_{2}=\varnothing$ in Theorem 1.2, we can both get the Theorem A.

\section{Notation and Preliminary Works}

For a compact subset $L$ of $\mathbb{R}^{n}$, with the origin in its interior, star-shaped with respect to the origin, the radial 
function $\rho(L, \cdot): S^{n-1} \rightarrow \mathbb{R}$, is defined by

$$
\rho(L, u)=\max \{\lambda: \lambda u \in L\} .
$$

If $\rho(L, \cdot)$ is continuous and positive, $L$ will be called a star body. Let $\varphi_{o}^{n}$ denote the set of star bodies in $\mathbb{R}^{n}$.

The mixed volume $V\left(K_{1}, \cdots, K_{n}\right)$ of the compact convex subsets $K_{1}, \cdots, K_{n}$ of $\mathbb{R}^{n}$ is defined by

$$
\begin{aligned}
& V\left(K_{1}, K_{2}, \cdots, K_{n}\right) \\
& =\frac{1}{n !} \sum_{j=1}^{n}(-1)^{n+j} \sum_{i_{1}<\cdots<i_{j}} V\left(K_{i_{1}}+K_{i_{2}}+\cdots+K_{i_{j}}\right) .
\end{aligned}
$$

If $K_{1}=\cdots=K_{n-i}=K, K_{n-i+1}=\cdots=K_{n}=L$, then $V\left(K_{1}, K_{2}, \cdots, K_{n}\right)$ will be denote as

$V_{i}(K, L)=V(K, n-i ; L, i)$. If $L=B_{n}$, then $V_{i}\left(K, B_{n}\right)$ is called the quermassintegrals of $K$; it will often be written as $W_{i}(K)(i=0,1, \cdots, n)$.

The mixed quermassintegrals

$W_{i}(K, L)(i=0,1, \cdots, n-1)$ of $K, L \in K^{n}$, are defined by [8]

$$
(n-i) W_{i}(K, L)=\lim _{\varepsilon \rightarrow 0^{+}} \frac{W_{i}(K+\varepsilon L)-W_{i}(K)}{\varepsilon},
$$

Since $W_{i}(\lambda K)=\lambda^{n-i} W_{i}(K)$, it follows that $W_{i}(K, K)=W_{i}(K)$, for all $i$. Since the quermassintegrals $W_{n-1}$ is Minkowski linear, it follows that $W_{n-1}(K, L)=W_{n-1}(L)$ for all $K$.

Aleksandrov [9] and Fenchel and Jessen [10] have shown that for $K \in \mathcal{K}^{n}$ and $i=0,1, \cdots, n-1$, there exists a regular Borel measure $S_{i}(K, \cdot)$ on $S^{n-1}$, such that the mixed quermassintegrals $W_{i}(K, L)$ has the following integral representation:

$$
W_{i}(K, L)=\frac{1}{n} \int_{S^{n-1}} h(L, u) \mathrm{d} S_{i}(K, u),
$$

for all $L \in \mathcal{K}^{n}$. The measure $S_{n-1}(K, \cdot)$ is independent of the body $K$ and is just ordinary Lebesgue measure, $S$ on $S^{n-1}$. The surface area measure $S_{0}(K, \cdot)$ will frequently be written simply as $S(K, \cdot)$.

Suppose $K, L \in \varphi_{o}^{n}, \lambda$ and $\mu$ are nonnegative real numbers and not both zero. To define the harmonic Blaschke addition, $\lambda K \hat{+} \mu L$, first define $\xi>0$ by [6]

$$
\begin{aligned}
\xi^{1 /(n+1)}= & \frac{1}{n} \int_{S^{n-1}}\left[\lambda V(K)^{-1} \rho(K, u)^{n+1}\right. \\
& \left.+\mu V(L)^{-1} \rho(L, u)^{n+1}\right]^{n /(n+1)} \mathrm{d} u .
\end{aligned}
$$

The body $\lambda K \hat{+} \mu L \in \varphi_{o}^{n}$ is defined as the body whose radial function is given by

$$
\begin{aligned}
& \xi^{-1} \rho(\lambda K \hat{+} \mu L, \cdot)^{n+1} \\
& =\lambda V(K)^{-1} \rho(K, \cdot)^{n+1}+\mu V(L)^{-1} \rho(L, \cdot)^{n+1} .
\end{aligned}
$$

\section{Inequalities for Centroid Body}

In this section, we will establish the inequality more general than Theorem 1.1 as follows.

Theorem 3.1. Let $K, L \in \varphi_{o}^{n}, \quad 0 \leq i<n-1$ and $0 \leq \alpha \leq 1$. Then

$$
\begin{aligned}
& W_{i}(\Gamma(K \hat{+} L))^{\frac{1}{n-i}} \\
\geq & W_{i}(\Gamma(\alpha K \hat{+}(1-\alpha) L))^{\frac{1}{n-i}} \\
& +W_{i}(\Gamma((1-\alpha) K \hat{+} \alpha L))^{\frac{1}{n-i}} \\
\geq & W_{i}(\Gamma K)^{\frac{1}{n-i}}+W_{i}(\Gamma L)^{\frac{1}{n-i}},
\end{aligned}
$$

with equality holds if and only if $\Gamma K$ and $\Gamma L$ are homothetic.

To prove Theorem 3.1, the following preliminary results will be needed:

Lemma 3.2. ([8]). Let $K, L \in \mathcal{K}^{n}$ and $0 \leq i<n-1$. Then

$$
W_{i}(K, L)^{n-i} \geq W_{i}(K)^{n-i-1} W_{i}(L),
$$

with equality if and only if $K$ and $L$ are homothetic.

Lemma 3.3. ([11]). Let $K, L \in \mathcal{K}^{n}, \quad 0 \leq i<n-1$. Then

$$
W_{i}(K+L)^{1 /(n-i)} \geq W_{i}(K)^{1 /(n-i)}+W_{i}(L)^{1 /(n-i)},
$$

with equality if and only if $K$ and $L$ are homothetic.

Proof of Theorem 3.1.

By (2.4), (2.5) and the polar coordinate formula for volume, we can get $\xi=V(K \hat{+} L)$. Hence from (2.5), we obtain

$$
\frac{\rho(\lambda K \hat{+} \mu L, \cdot)^{n+1}}{V(\lambda K \hat{+} \mu L)}=\frac{\lambda \rho(K, \cdot)^{n+1}}{V(K)}+\frac{\mu \rho(L, \cdot)^{n+1}}{V(L)} .
$$

Using polar coordinates, (1.2) can be written as an integral over $S^{n-1}$

$$
h(\Gamma K, u)=\frac{1}{V(K)} \int_{S^{n-1}}|u \cdot v| \rho_{K}(v)^{n+1} \mathrm{~d} v .
$$

Then from (3.3) and (3.4), we have

$$
h(\Gamma(\lambda K \hat{+} \mu L), u)=\lambda h(\Gamma K, u)+\mu h(\Gamma L, u) .
$$

For $K, L \in \varphi_{o}^{n}$ and $0 \leq \alpha \leq 1$. Let

$$
\begin{aligned}
& F=\Gamma(\alpha K \hat{+}(1-\alpha) L), \\
& G=\Gamma((1-\alpha) K \hat{+} \alpha L),
\end{aligned}
$$

By (2.3) and (3.5), we have 


$$
\begin{aligned}
W_{i}(\Gamma(K \hat{+} L)) & =\frac{1}{n} \int_{S^{n-1}} h(\Gamma(K \hat{+} L), u) \mathrm{d} S_{i}(\Gamma(K \hat{+} L), u)=\frac{1}{n} \int_{S^{n-1}}[h(\Gamma K, u)+h(\Gamma L, u)] \mathrm{d} S_{i}(\Gamma(K \hat{+} L), u) \\
& =\frac{1}{n} \int_{S^{n-1}}[\alpha h(\Gamma K, u)+(1-\alpha) h(\Gamma L, u)+(1-\alpha) h(\Gamma K, u)+\alpha h(\Gamma L, u)] \mathrm{d} S_{i}(\Gamma(K \hat{+} L), u) \\
& =\frac{1}{n} \int_{S^{n-1}}[h(\Gamma(\alpha K \hat{+}(1-\alpha) L), u)+h(\Gamma((1-\alpha) K \hat{+} \alpha L), u)] \mathrm{d} S_{i}(\Gamma(K \hat{+} L), u) \\
& =\frac{1}{n} \int_{S^{n-1}}[h(F, u)+h(G, u)] \mathrm{d} S_{i}(\Gamma(K \hat{+} L), u)=\frac{1}{n} \int_{S^{n-1}} h(F+G, u) \mathrm{d} S_{i}(\Gamma(K \hat{+} L), u) .
\end{aligned}
$$

That is

$$
W_{i}(\Gamma(K \hat{+} L))=W_{i}(\Gamma(K \hat{+} L), F+G) .
$$

By Lemma 3.2, we get

$$
W_{i}(\Gamma(K \hat{+} L)) \geq W_{i}(K \hat{+} L)^{(n-i-1) /(n-i)} W_{i}(F+G)^{1 /(n-i)},
$$

which implies that,

$$
W_{i}(\Gamma(K \hat{+} L)) \geq W_{i}(F+G),
$$

with equality holds if and only if $\Gamma(K \hat{+} L)$ and $F+G$ are homothetic.

The Brunn-Minkowski inequality (3.2) can now be used to conclude that

$$
W_{i}(F+G)^{1 /(n-i)} \geq W_{i}(F)^{1 /(n-i)}+W_{i}(G)^{1 /(n-i)},
$$

with equality holds if and only if $F$ and $G$ are homothetic.

By (3.7) and (3.8), we get the first inequality of Theorem 3.1. By the equality conditions of (3.7) and (3.8), the first equality of Theorem 3.1 holds if and only if $\Gamma K$ and $\Gamma L$ are homothetic.

By (3.5) and Lemma 3.3, we get

$$
\begin{aligned}
& W_{i}(F)^{1 /(n-i)}=W_{i}(\Gamma(\alpha K \hat{+}(1-\alpha)))^{1 /(n-i)} \\
& =W_{i}(\alpha \Gamma K+(1-\alpha) \Gamma L)^{1 /(n-i)} \\
& \geq \alpha W_{i}(\Gamma K)^{1 /(n-i)}+(1-\alpha) W_{i}(\Gamma L)^{1 /(n-i)},
\end{aligned}
$$

Similarly,

$$
W_{i}(G)^{1 /(n-i)} \geq(1-\alpha) W_{i}(\Gamma K)^{1 /(n-i)}+\alpha W_{i}(\Gamma L)^{1 /(n-i)} .
$$

Hence,

$$
\begin{aligned}
& W_{i}(F)^{1 /(n-i)}+W_{i}(G)^{1 /(n-i)} \\
& \geq W_{i}(\Gamma K)^{1 /(n-i)}+W_{i}(\Gamma L)^{1 /(n-i)},
\end{aligned}
$$

with equality holds if and only if $\Gamma K$ and $\Gamma L$ are homothetic. This completes the proof.

Let $i=0$ in Theorem 3.1, we obtain an isolate form of Brunn-Minkowski inequality for centroid body.

Corollary 3.4. Let $K, L$ be star bodies in $\mathbb{R}^{n}$ and $0 \leq \alpha \leq 1$.

$$
\begin{aligned}
& V(\Gamma(K \hat{+} L))^{\frac{1}{n}} \\
& \geq V(\Gamma(\alpha K \hat{+}(1-\alpha) L))^{\frac{1}{n}}+V(\Gamma((1-\alpha) K \hat{+} \alpha L))^{\frac{1}{n}} \\
& \geq V(\Gamma K)^{\frac{1}{n}}+V(\Gamma L)^{\frac{1}{n}},
\end{aligned}
$$

the equality holds if and only if $\Gamma K$ and $\Gamma L$ are homothetic.

Now, we establish the volume difference of BrunnMinkowski inequality for centroid body.

Theorem 3.5. Let $K$ and $L$ be star bodies in $\mathbb{R}^{n}$. Ellipsoid $E_{1} \subset K$, and $E_{2} \subset L$ is a homothetic copy of $E_{1}$. Then

$$
\begin{aligned}
& {\left[V(\Gamma(K \hat{+} L))-V\left(\Gamma\left(E_{1} \hat{+} E_{2}\right)\right)\right]^{\frac{1}{n}}} \\
& \geq\left[V(\Gamma K)-V\left(\Gamma E_{1}\right)\right]^{\frac{1}{n}}+\left[V(\Gamma L)-V\left(\Gamma E_{2}\right)\right]^{\frac{1}{n}},
\end{aligned}
$$

the equality holds if and only if $\Gamma K$ and $\Gamma L$ are homothetic and

$$
\left(V(\Gamma K), V\left(\Gamma E_{1}\right)\right)=\mu\left(V(\Gamma L), V\left(\Gamma E_{2}\right)\right),
$$

where $\mu$ is a constant.

To prove Theorem 3.5, we need the following two lemmas:

Lemma 3.6. (Bellman's inequality) ([12], p. 38). Suppose that $a=\left\{a_{1}, a_{2}, \cdots, a_{n}\right\}$ and $b=\left\{b_{1}, b_{2}, \cdots, b_{n}\right\}$ are two n-tuples of positive real numbers, and $p>1$ such that

$$
a_{1}^{p}-\sum_{i=2}^{n} a_{i}^{p}>0 \text { and } b_{1}^{p}-\sum_{i=2}^{n} b_{i}^{p}>0 .
$$

Then

$$
\begin{aligned}
& \left(\left(a_{1}+b_{1}\right)^{p}-\sum_{i=2}^{n}\left(a_{i}+b_{i}\right)^{p}\right)^{\frac{1}{p}} \\
& \geq\left(a_{1}^{p}-\sum_{i=2}^{n} a_{i}^{p}\right)^{\frac{1}{p}}+\left(b_{1}^{p}-\sum_{i=2}^{n} b_{i}^{p}\right)^{\frac{1}{p}},
\end{aligned}
$$

with equality if and only if $a=v b$, where $v$ is $a$ constant. 
Lemma 3.7. (Busemann-Petty centroid inequality) ([4], p. 359). Let $K \in \mathcal{K}^{n}$. Then

$$
V(\Gamma K) \geq\left(\frac{2 k_{n-1}}{(n+1) k_{n}}\right)^{n} V(K),
$$

with equality if and only if $K$ is a centered ellipsoid.

Proof of Theorem 1.2. Applying inequality (1.3), we have

$$
V(\Gamma(K \hat{+} L))^{\frac{1}{n}} \geq V(\Gamma K)^{\frac{1}{n}}+V(\Gamma L)^{\frac{1}{n}},
$$

the equality holds if and only if $\Gamma K$ and $\Gamma L$ are homothetic.

$$
V\left(\Gamma\left(E_{1} \hat{+} E_{2}\right)\right)^{\frac{1}{n}}=V\left(\Gamma E_{1}\right)^{\frac{1}{n}}+V\left(\Gamma E_{2}\right)^{\frac{1}{n}},
$$

From (3.9) and (3.10), we obtain that

$$
\begin{aligned}
& V(\Gamma(K \hat{+} L))-V\left(\Gamma\left(E_{1} \hat{+} E_{2}\right)\right) \\
& \geq\left[V(\Gamma K)^{\frac{1}{n}}+V(\Gamma L)^{\frac{1}{n}}\right]^{n}-\left[V\left(\Gamma E_{1}\right)^{\frac{1}{n}}+V\left(\Gamma E_{2}\right)^{\frac{1}{n}}\right]^{n} .
\end{aligned}
$$

Since $E_{1} \subset K$ and $E_{2} \subset L$, by Lemma 3.7, we get

$$
\begin{aligned}
V(\Gamma K) & \geq\left(\frac{2 k_{n-1}}{(n+1) k_{n}}\right)^{n} V(K) \\
& >\left(\frac{2 k_{n-1}}{(n+1) k_{n}}\right)^{n} V\left(E_{1}\right)=V\left(\Gamma E_{1}\right),
\end{aligned}
$$

and

$$
V(\Gamma L)>V\left(\Gamma E_{2}\right),
$$

By (3.11) and Bellman's inequality, we get

$$
\begin{aligned}
& {\left[V(\Gamma(K \hat{+} L))-V\left(\Gamma\left(E_{1} \hat{+} E_{2}\right)\right)\right]^{\frac{1}{n}}} \\
& \geq\left[V(\Gamma K)-V\left(\Gamma E_{1}\right)\right]^{\frac{1}{n}}+\left[V(\Gamma L)-V\left(\Gamma E_{2}\right)\right]^{\frac{1}{n}} .
\end{aligned}
$$

By the equality conditions of (3.9) and the Bellman's inequality, the equality of (3.12) holds if and only if $\Gamma K$ and $\Gamma L$ are homothetic and

$$
\left(V(\Gamma K), V\left(\Gamma D_{1}\right)\right)=\mu\left(V(\Gamma L), V\left(\Gamma D_{2}\right)\right),
$$

where $\mu$ is a constant. This completes the proof.

\section{Acknowledgements}

The authors would like to acknowledge the support from the National Natural Science Foundation of China (11101216, 11161024), Qing Lan Project and the Nanjing Xiaozhuang University (2009XZRC05, 2010KYQN24).

\section{REFERENCES}

[1] J. Yuan, L. Z. Zhao and G. S. Leng, "Inequalities for $L_{p}$ Centroid Body,” Taiwanese Journal of Mathematics, Vol. 11, No. 5, 2007, pp. 1315-1325.

[2] C. M. Petty, “Centroid Surface,” Pacific Journal of Mathematics, Vol. 11, No. 4, 1961, pp. 1535-1547. doi:10.2140/pjm.1961.11.1535

[3] K. Leichtwei, “Affine Geometry of Convex Bodies,” J. A. Barth, Heidelberg, 1998.

[4] R. Schneider, "Convex Bodies: The Brunn-Minkowski Theory,” Cambridge University Press, Cambridge, 1993. doi:10.1017/CBO9780511526282

[5] R. J. Gardner, “Geometric Tomography,” Cambridge University Press, Cambridge, 1995.

[6] E. Lutwak, "Centroid Bodies and Dual Mixed Volumes," Proceedings London Mathematical Society, Vol. 60, No. 2, 1990, pp. 365-391. doi:10.1112/plms/s3-60.2.365

[7] G. Y. Zhang, "Centered Bodies and Dual Mixed Volumes," Transactions of the American Mathematical Society, Vol. 345, No. 2, 1994, pp. 777-801. doi:10.1090/S0002-9947-1994-1254193-9

[8] E. Lutwak, "The Brunn-Minkowski-Firey Theory I: Mixed Volumes and the Minkowski Problem,” Journal of Differential Geometry, Vol. 38, 1993, pp. 131-150.

[9] A. D. Aleksandrov, "Zur Theorie der Gemischten Volumina von Konvexen Körpern, I. Verallgemeinerung einiger Begriffe der Theorie der Konvexen Körpern,” Matematicheskii Sbornik, Vol. 2, 1937, pp. 947-972.

[10] W. Fenchel and B. Jessen, "Mengenfunktionen und Konvexe Körpern,” Danske Videnskabernes Selskab. Matematisk-Fysiske Meddelelser, Vol. 16, 1938, p. 3.

[11] R. J. Gardner, “The Brunn-Minkowski Inequality,” Bulletin of the American Mathematical Society, Vol. 39, No. 3, 2002, pp. 355-405. doi:10.1090/S0273-0979-02-00941-2

[12] E. F. Beckenbach and R. Bellman, "Inequalities,” Springer, Berlin, 1961. doi:10.1007/978-3-642-64971-4 